\title{
Prospective multicentre study on the evaluation of antituberculosis treatment results in Italy: comparison of the culture- versus the smear-based methods
}

\author{
G.B. Migliori*, M. Ambrosetti*\$ ${ }^{\S}$ G. Besozzi ${ }^{+}$, L. Casali ${ }^{\#}$, M.C. Raviglione ${ }^{*}$ and the National AIPO \\ "Tuberculosis" Study Group
}

Prospective multicentre study on the evaluation of antituberculosis treatment results in Italy: comparison of the culture-versus the smear-based methods. G.B. Migliori, M. Ambrosetti, G. Besozzi, L. Casali, M.C. Raviglione, and the National AIPO "Tuberculosis" Study Group. (C)ERS Journals Ltd 1999.

ABSTRACT: Cohort analysis of treatment outcomes is the most informative technique to evaluate the tuberculosis (TB) control programme. The aim of the study was to assess treatment outcomes comparing the smear- versus the culture-based methods, using data on TB patients treated under programme conditions in Italy.

This was a prospective monitoring study based on the standardized collection of forms from a representative sample of Italian TB Units. The forms, with individual data, were reviewed and analysed on a quarterly basis according to the principles of cohort analysis, using both the smear- and culture-based methods. The complete bacteriological profile of patients was analysed at diagnosis and at completion of treatment.

Nine hundred and ninety-two TB cases were notified. Among 681 pulmonary cases, 368 cases were culture-confirmed at diagnosis $(333$ new and 35 retreatment cases, 293 being sputum smear positive, $79.6 \%$ ). At the end of treatment, out of the 333 new culture-confirmed cases, $136(\mathbf{4 0 . 8 \%})$ were defined "cured" using the culture-based method and $108(32.4 \%)$ using the smear-based method $\left(p<0.05, \chi^{2}\right.$ test).

The culture-based method is the recommended tool to evaluate pulmonary tuberculosis treatment results. Culture allows a more precise definition of a "cured" patient in both sputum smear positive and negative tuberculosis cases.

Eur Respir J 1999; 13: 900-903.
*Fondazione S. Maugeri, Clinica del Lavoro e della Riabilitazione, Care and Research Institute, Tradate, Italy. ${ }^{5}$ Postgraduate School of Internal Medicine, Varese University, Italy. ${ }^{+}$Villa Marelli Institute/National Reference Laboratory, Milan, Italy. "Dept of Pulmonary Diseases, Perugia University, Italy. "Global Tuberculosis Programme, World Health Organization, Geneva, Switzerland

Correspondence: G.B. Migliori, Fondazione Salvatore Maugeri, Clinica del Lavoro e della Riabilitazione, Care and Research Institute, via Roncaccio 16, 21049 Tradate (VA), Italy, Fax: 390331829133

Keywords: Culture

methodology

sputum smear

tuberculosis

treatment outcomes

Received: February 171998

Accepted after revision December 141998

Funded by the I and II TB Projects, Istituto Superiore di Sanitá, Rome, Grant $\mathrm{N}^{\circ} 1$, $641 / 96-4,857 / 97$ and 6,523/97
The objective of tuberculosis (TB) programmes is the elimination of TB from the society by stopping the transmission of TB infection [1-3]. It can be achieved through the rapid identification and effective treatment of infectious cases [1-3]. For programme planning and evaluation, it is necessary that adequate data on case findings and treatment results are collected and analysed systematically to quantify performance and its trend [4]. Therefore, cohort analysis of treatment outcomes is the most informative technique for evaluating the treatment programme [4]. The World Health Organization (WHO) and the International Union Against Tuberculosis and Lung Disease (IUATLD) recommend a method (focused on sputum smear results) to evaluate treatment outcomes in countries with a high incidence of TB based on six mutually exclusive categories (cure, treatment completion, death, failure, treatment interruption, transfer out) $[4,5]$. Recently, an adaptation of this system of treatment outcome monitoring, based on culture rather than smear results, has been proposed for Europe by the WHO and IUATLD [6, 7]. At present, no study is available in the literature which describes the methodological problems arising from cohort analysis of treatment results performed in countries with a low incidence of TB under programme conditions.

The aim of the present study was to assess treatment outcomes in a low incidence country comparing the smearversus culture-based methods, using data from TB patients treated under programme conditions in Italy.

\section{Methods}

This was a prospective monitoring study based on the collection of standard recording and reporting forms from a representative sample of Italian TB units $(n=41)$ covering about $21 \%$ of all TB cases notified every year in Italy [8].

On a quarterly basis, 9 months after enrollment, the participating units sent individual notification forms to both the co-ordinating centre (Tradate) and three area supervisors. Data forms, after the revision process, were stored in a database at the co-ordinating centre, using Powersoft Powerbuilder 5.0 (Powersoft, Concorde, MD, USA). The participating units were monitored on a monthly basis by the area supervisors and, if necessary, by the co-ordinating 
unit staff. All mistakes and inconsistencies identified in the forms were corrected during the monitoring visits. The TB Units adopted the WHO recommended standardized short course chemotherapy regimens (i.e. for new cases two months of rifampicin, isoniazid, ethambutol and pyrazinamide followed by 4 months of isoniazid and rifampicin) [8-10]. All the definitions used were derived from published recommendations of the WHO $[1,3,4-7,10]$. In particular, the patients were defined as: "new case", those who never had treatment for TB or who had taken anti-TB drugs for $<4$ weeks [10]; "retreatment case", those who had taken anti-TB drugs for $>4$ weeks; and "definite case", those with culture confirmed disease due to Mycobacterium tuberculosis complex [6]. According to the smearbased method, a patient is defined "cured" if sputum smears are negative on two occasions at the end of the treatment, and "treatment completed" if there is documented treatment completion, but no sputum smear microscopy at the end of the treatment. According to the culturebased method, a patient is defined "cured" in the presence of a documented culture conversion during the continuation phase, and "treatment completed" when treatment completion (in the absence of evidence for culture conversion) is documented. "Treatment success" is defined as the sum of patients whose outcome was "cured" and "treatment completed" divided by the total number of cases registered for treatment and expressed as a percentage [7].

Treatment outcomes were reported individually. The denominator for all treatment outcomes was the total number of consecutive patients registered for treatment between January 1, 1995 and March 31, $1996(n=992)$ [5, 7]. Treatment outcomes were stratified into new and retreatment cases, and into pulmonary (sputum smear positive (SSP) and negative (SSN)) and extrapulmonary cases (EP) [10]. Out of all pulmonary cases $(n=681)$, a core data set of culture confirmed pulmonary TB cases was identified for further analysis $(n=368)$. Within this data set, treatment outcomes were assessed using both the smearbased [5] and culture-based methods [7]. The denominator was the total number of consecutive culture positive pul- monary patients registered for treatment in the study period. As it was expected that a certain proportion of cases would have no bacteriological monitoring at the end of treatment, the individual form included a field for information on reasons for lack of bacteriological confirmation at the end of treatment. An additional analysis was performed to obtain further information on the issue.

Observed and expected frequencies were compared by means of $2 \times 2$ contingency tables using uncorrected Chisquared test. A p-value $<0.05$ was considered statistically significant.

\section{Results}

\section{TB cases notified}

Out of 992 TB cases notified, 897 (90.4\%) were new cases and $95(9.6 \%)$ were retreatment cases. The bacteriological confirmation of these cases is shown in figure 1. No significant difference with national data was found for the relevant variables available (bacteriologically confirmed cases, age and sex distribution of cases).

\section{New definite pulmonary cases}

Out of 368 definite pulmonary cases, $333(90.5 \%)$ were new cases. Among them, $265(79.6 \%)$ were SSP, 51 $(15.3 \%)$ were SSN and $17(5.1 \%)$ were not tested by sputum smear at diagnosis. At the end of treatment, 139 $(41.7 \%)$ out of all 333 new definite pulmonary cases were tested by culture and $136(97.8 \%)$ of them were culture negative. Out of 265 new definite pulmonary SSP cases, sputum smear examination at the end of treatment was performed in $114(43 \%)$ cases, $109(95.6 \%)$ of them being SSN. Treatment outcome of new definite pulmonary cases is summarized in figure 2. Out of 333 new definite pulmonary cases, $136(40.8 \%)$ were defined "cured" using the culture-based method and 108 (32.4\%) using the

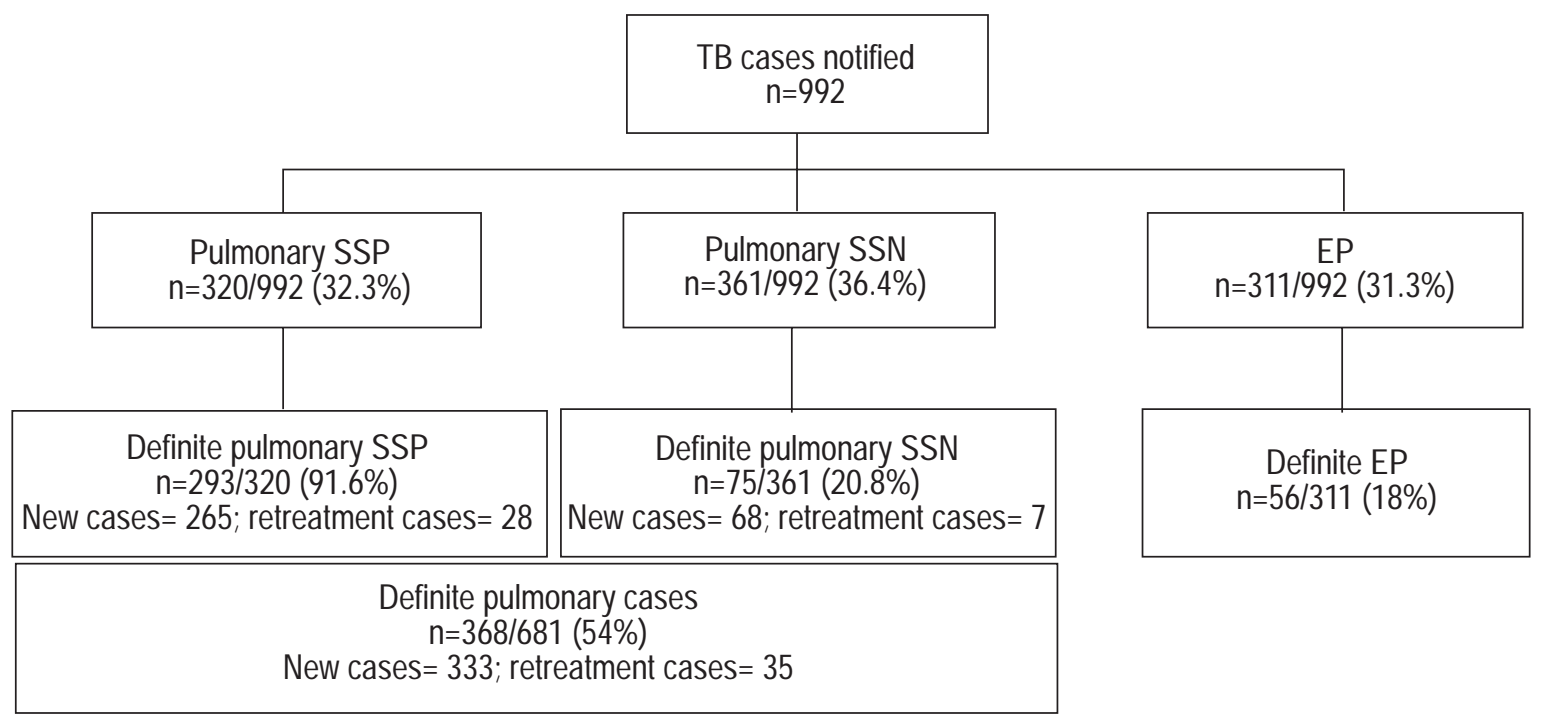

Fig. 1. - Bacteriological confirmation of 992 tuberculosis (TB) cases notified. SSP: sputum smear positive; SSN: sputum smear negative; EP: extrapulmonary; Definite cases: culture confirmed cases. 


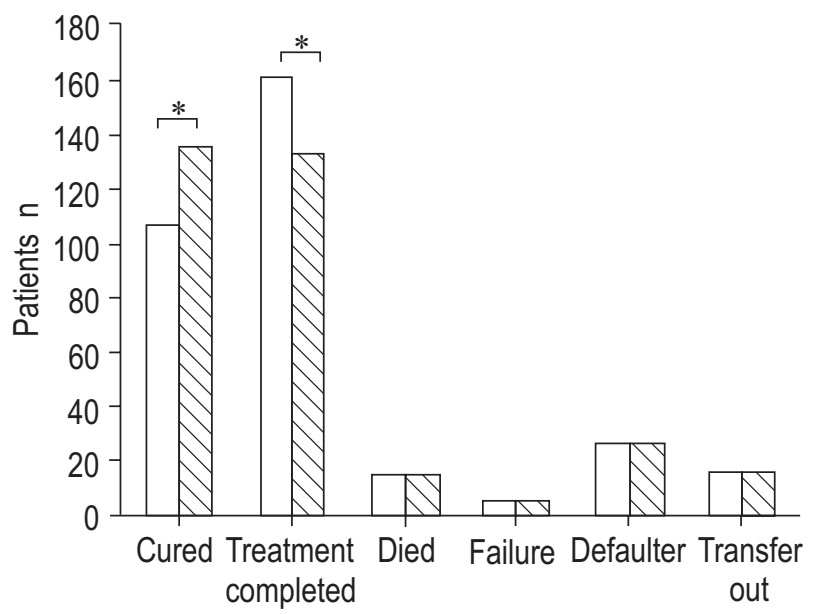

Fig. 2. - Treatment outcomes of 333 new definite pulmonary tuberculosis cases based on smear $(\square)$ and culture $(\mathbb{\nabla})$ results. ${ }^{*}: \mathrm{p}<0.05$, uncorrected $\chi^{2}$ test.

smear-based method ( $p<0.05, \chi^{2}$-test). Among SSN cases, a radiological improvement at the end of treatment was reported in $83.3 \%$ of them.

\section{Retreatment definite pulmonary cases}

Out of 368 definite pulmonary cases, 35 (9.5\%) were retreatment cases. Among them, $28(80 \%)$ were SSP, 3 $(8.6 \%) \mathrm{SSN}$ and $4(11.4 \%)$ not tested by sputum smear at diagnosis. At the end of treatment, $10(28.6 \%)$ out of all retreatment definite pulmonary cases were tested by culture and $8(80 \%)$ of them were culture negative. Out of 28 retreatment definite pulmonary SSP cases, sputum smear examination at the end of treatment was performed in 9 $(32.1 \%)$ cases, $7(77.7 \%)$ of them being SSN. Treatment success was achieved in $18(51.4 \%)$ cases, while $7(20 \%)$ cases died, $2(5.7 \%)$ failed and $8(22.9 \%)$ defaulted. No significant difference was found comparing cases "cured" obtained by the smear-based and the culture-based method (7 (20\%) versus 8 (22.9\%) respectively; NS).

\section{Reasons for absence of sputum result at end of treat- ment}

As no compulsory report of comments at the end of treatment was established, the reason for absence of sputum result was available in 83 cases out of $170(49 \%)$ : in 13 of them the laboratory did not stain the sputum because it was deemed as saliva, while in 70 cases the patient was unable to produce sputum because of the clinical improvement.

\section{Discussion}

According to the WHO, high cure rate, low level of acquired drug resistance and high detection rate define an effective TB control programme [10]. As a high cure rate of infectious patients is the main target of TB control, the evaluation of treatment outcomes is a relevant indicator of programme performance $[4,7]$. Although data on treatment results from high incidence countries are often available, few industrialized low incidence countries in Europe (e.g. the Netherlands, the Czech Republic and Slovakia) have implemented such a procedure [11, 12]). The methodology to evaluate treatment outcomes through cohort analysis has been validated in high incidence countries, focusing on smear results $[4,5]$. The present study compared treatment outcomes of TB patients in a low incidence country applying the smear- versus culturebased methods with the aim of defining which of the two methods is more reliable. No significant difference was found in treatment outcomes according to the smearversus culture-based methods for SSP cases. A significant difference was found for SSN cases, which, by definition, cannot be defined as cured (but only treatment completed).

Under a public health perspective, a methodology including a double cohort analysis (sputum smear- and culture-based) of culture confirmed cases may be proposed in low incidence countries, allowing the comparison of treatment outcomes among "definite" cases in these countries with those of high incidence countries (where the available smear-based approach focuses on infectious cases). Under the clinician's perspective, treatment monitoring is important in patients undergoing anti-TB chemotherapy in order to evaluate the response to treatment and rapidly identify and manage drug-induced toxicity [10]. Bacteriological monitoring is possible only in patients who are culture positive and/or SSP. Using the smear-based method one should rely on sputum smear results, performed at the end of the intensive phase of treatment ( 2 nd month for new cases) and at the end of treatment (5th and 6th month for new cases). Using the culture-based method, one has the option to rely on culture only or to use sputum smear to obtain additional (and readily available) information. According to the proposed definitions, a "definite" case is defined cured when culture conversion is achieved during the continuation phase [7]. While a negative sputum smear after two months of treatment in a definite case allows the clinician to wait for the final culture, an SSP result suggests the need for medical action (to prolong the intensive phase, to ask for a culture, etc.).

In addition the results of this study show that, in Italy: 1) only $54 \%$ of pulmonary TB cases are culture confirmed at diagnosis $(368 / 681)$ and only $21.9 \%$ monitored by culture at the end of treatment $(149 / 681)$; 2) in a relevant proportion of cases, the sputum was not performed at the end of treatment because the patient was unable to produce sputum or because a salivary sample was obtained; and 3) the proportion of patients other than SSP (SSN and sputum not done) is higher than expected $(388 / 681,57 \%)$. The observation that 83 pulmonary patients SSP and/or culture positive at diagnosis were not monitored at the end of treatment because of lack of sputum suggests that, in Europe, according to the present definitions [8], a relevant proportion of patients being really "cured" might be classified as treatment completed $[6,8]$.

The results of this study underline the importance of further disseminating guidelines among physicians diagnosing, reporting and treating tuberculosis at national level and within scientific societies. The proportion of bacteriologically unconfirmed patients ("other than definite cases" [6]) might reflect early diagnosis (cases with a negative bacteriological examination), or for patients without bacteriological examination performed, a pitfall of the programme (overemphasis on chest radiographs and clinical 
presentation for diagnosis) and the difficulty of obtaining sputum samples (cases unable to produce sputum or merely producing saliva). Based on the consideration that some cases diagnosed as "minimal tuberculosis" (sputum smear negative) might be affected by diseases other than tuberculosis, the final and more reliable analysis was performed on culture confirmed cases only, as recommended by a World Health Organization/International Union Against Tuberculosis and Lung Disease task force [7].

Members of the National AIPO "Tuberculosis" Study Group. Chairman: L. Casali (Perugia). Secretary: G.B. Migliori (Tradate). Coordinators: G. Besozzi (Milan), R. Le Donne (Rieti), G. Montesano (Matera). Supervisor: G. Di Pisa (Sondalo). Members: G. Agati (Reggio di Calabria), S. Aiolfi (Crema), A. Altieri (Rome), M. Ambrosetti (Tradate), W. Arossa (Turin), G. Bagnasco (Turin), G. Bazzerla (Vittorio Veneto), A. Berra (Salerno), M. Bugiani (Turin), S. Calabro (Feltre). G. Castiglioni (Tradate), M. Cavallero (Turin), F. Cisno (Feltre), L.R. Codecasa (Milan), V. Colorizio (L'Aquila), M. Confalonieri (Piacenza), L. D'Ambrosio (Tradate), V. D'Ambrosio (Gallarate), M. Ermeti (Rimini), E. Faccini (Treviso), G. Farinelli (Rieti), R. Fatigante (Potenza), B. Farris (Cagliari), T. Fatur Volante (Como), G. Felisatti (Ferrara), F. Fiorentini (Vecchiazzano), P. Ferranti (Terni), C. Foschi (Cesena), A. Garzone (Novara), F. Gozzelino (Biella), L. Jacopino (Reggio di Calabria), G. Jeni (Messina), G. Lauriello (Salerno), G.P. Ligia (Cagliari), R. Longi (Rimini), G. Macor (Pinerolo), L. Manca (Nuoro), D. Mancini (Rieti), F. Marchesani (Cremona), S. Marcias (Oristano), A. Monaco (Perugia), S. Nardini (Vittorio Veneto), S. Nutini (Florence), G. Orani (Cagliari), M. Parpanesi (Cremona), A. Pasi (Pavia), O. Penza (Perugia), L. Petrozzi (Bari), P. Pretto (Bolzano), S. Rossi (Terni), P. Ramorino (Genoa), M.C. Raviglione (Geneva, Switzerland), E. Sabato (Brindisi), L. Saini (Novara), G. Selva (Biella), A. Spanevello (Tradate), R. Tazza (Terni), G. Trucco (Imperia), U. Viviani (Ferrara), M. Volante (Como), S. Viola (Rho), D. Volpes (Palermo), F. Zaccara (Rho).

\section{References}

1. Clancy L, Rieder HL, Enarson DA, Spinaci S. Tuberculosis elimination in the countries of Europe and other industrialized countries. Eur Respir $J$ 1991; 4: 12881295.

2. Enarson DA. The International Union Against Tuberculosis and Lung Disease model National Tuberculosis Programmes. Tuberc Lung Dis 1995; 76: 95-99.

3. World Health Organization. WHO Framework for Effective Tuberculosis Control. Geneva, WHO/TB/94. 179, 1994; pp. 1-13.

4. World Health Organization, Tuberculosis Unit, Division of Communicable Diseases. Tuberculosis Surveillance and Monitoring. Geneva, WHO/TB/91. 163, 1991; pp. 118.

5. World Health Organization. Managing tuberculosis at District Level. A Training Course. Module A5: Monitoring Treatment. Geneva, WHO, L 1994; pp. 1-68.

6. Rieder HL, Watson JM, Raviglione MC, et al. Surveillance of tuberculosis in Europe. Eur Respir J 1996; 9: 1097-1104.

7. Veen J, Raviglione MC, Rieder HL, et al. Standardized treatment outcome monitoring in Europe. Recommendations of Working Group of WHO and IUATLD. Eur Respir J 1998; 12: 505-510.

8. Migliori GB, Ambrosetti M, Besozzi G, et al. A costeffectiveness analysis of different policies of management of tuberculosis patients in Italy. Bull World Health Organ 1999; (in press).

9. Migliori GB, Casali L, Nardini S, et al. Evaluation of the impact of guidelines on tuberculosis control in Italy. Monaldi Arch Chest Dis 1996; 51: 204-209.

10. World Health Organization. Treatment of tuberculosis. Guidelines for National Programmes. Geneva, WHO/TB/ 97.220, 1997; pp. 1-77.

11. World Health Organization, Global Tuberculosis Programme. Global Tuberculosis Control. WHO Report 1998. Geneva, WHO/TB/98. 237, 1998; pp. 1-163.

12. Veen J, Kalisvaart NA. Index Tuberculosis 1995, The Netherlands. The Hague, Royal Netherlands Tuberculosis Association (KNCV). 1996; pp. 1-100. 